original papers

MERVYN LONDON, JUAN CANITROT, ADRIAN DZIALDOWSKI, ROBERT BATES

AND ALAN GWYNN

\title{
Contact with treatment services among arrested drug
} users

\author{
AIMS AND METHOD \\ To identify the proportion of \\ arrested drug misusers with prior \\ contact with treatment services, \\ police surgeons' records were exam- \\ ined over a 12-month period and \\ compared with the records of the \\ local drug misuse services. Both \\ referrals and those who attended \\ with face-to-face contact were \\ noted.
}

\author{
RESULTS \\ Sixty-seven per cent of drug-related \\ cases had been referred to services \\ prior to arrest and $58 \%$ had attended \\ with face-to-face contact on at least \\ one occasion. Homeless and male \\ drug users were more likely to have \\ had no past contact. Police surgeons \\ treated three-quarters of the cases \\ for opiate dependence. Drug mis- \\ users were much more likely to have \\ had past contact with services than \\ arrested alcohol misusers, and were \\ more likely to reoffend.
}

\begin{abstract}
CLINICAL IMPLICATIONS
The evidence base for motivational enhancement in the transient coercive setting of police custody is not established, and arrest referral schemes might not be cost-effective in areas where most cases have had previous contact with services. Local research may contribute to more informed decisions about these treatment-related criminal justice initiatives.
\end{abstract}

Arrest referral schemes to divert drug misusers from the criminal justice system into treatment are being established across the UK. Detention in a police station might encourage some drug users to reappraise their situation and become more amenable to the idea of treatment. It might be expected that any intervention at the time of arrest would have a greater impact on those with no previous contact with services. Providing information on where to find help to those who lack this knowledge may be very cost-effective and thus facilitate referrals. However, if a large number of cases have had prior contact with services, the intervention would have to enhance motivation significantly to be effective. Those who have dropped out of services in the past would have to be persuaded to re-engage in treatment. The evidence base for the efficacy of such motivational enhancement in the context of temporary detention is unclear.

Pearson et al (2000) showed that $65 \%$ of opiate users detained in London police stations were seen by a police surgeon. An evaluation of three arrest referral schemes found that $70 \%$ of arrested opiate users were seen by drug workers (Edmunds, 1998; http://www.dpas.gov.uk/cdpur23.pdf). Police surgeons may therefore have a similar level of contact with this target group, particularly as they provide 24-hour cover to police stations. This study seeks to identify the proportion of people arrested with substance misuse problems who have had no contact with treatment services by examining police surgeons' records. It was carried out before the introduction of an arrest referral scheme.

Cambridge, like other centres, has a sizeable drug misuse problem. Bennett (1998; http:// www.homeoffice.gov.uk/rds/pdfs/hors/83.pdf) found that $66 \%$ of those arrested in Cambridge tested positive for illegal drugs in their urine. Across five English cities, the average was $68 \%$. One in five of those arrested in Cambridge was positive for opiates. When this study was carried out, there was open access to the specialist drug treatment and advice services in Cambridge. Drug users could refer themselves directly for treatment and did not have to be seen by a general practitioner.

\section{Method}

Two police surgeons' medical records were examined for all cases related to drugs and alcohol seen during a 12month period. Demographic data, self-reported recent substance use, information on clinical interventions and recorded diagnoses were collected. Any requests for the police surgeon to test blood alcohol levels were not included.

The records of both local drug and alcohol treatment and advice services in Cambridge were then examined. One is a community drug team offering a prescribing service, while the other is a drop-in street agency providing information and advice but no prescriptions. Information was collected on all contacts with these services before and up to 3 months after arrest. The type of contact with services was also noted. Those who made face-to-face contact at least once prior to arrest were identified as a subgroup for further analysis. This group clearly knew how to access treatment, but this assumption could not be made for the rest of the sample.

\section{Results}

Of the 145 cases identified, 17 did not live locally and for a further four cases initially included in the study, insufficient data were available. The investigated sample thus consisted of 124 cases. Of these, 92 were 
drug-related and 32 alcohol-related cases. Sixty-two (67\%) of the drug-related cases had been referred to specialist drug-treatment services prior to arrest and 53 cases $(58 \%)$ were seen face-to-face on at least one occasion. The proportion of alcohol cases seen prior to arrest was much lower (31\%). During the 3 months after arrest, 17 drug-related cases were seen - one of whom was entirely new to the service and another who had been referred, but had not attended.

The average age of the 92 drug-related cases was 28 years and $27 \%$ were women. The 53 drug-related cases with face-to-face contact prior to arrest were compared with the remaining 39 drug misusers. Women were significantly more likely to have been seen, making up $42 \%$ of this face-to-face contact group and only $8 \%$ of the rest $(P=0.003)$. Homelessness and residence in temporary accommodation such as hostels were more common in those without previous face-to-face contact. Two-thirds of those with such contact lived in stable accommodation, compared with only $46 \%$ of those not seen $(P=0.05)$. Of the 92 cases, only $18 \%$ were prescribed no medication. Seventy-five per cent were prescribed opiates, mostly as a combination of dihydrocodeine and diazepam. The remaining $7 \%$ received only benzodiazepines.

The police surgeons' diagnosis was documented in 81 cases: 11 cases were noted to be intoxicated from opiates, 52 were withdrawing, six were poly-drug users and 12 had a non-specific opiate-related diagnosis. Selfreported drug use was documented in 82 cases. Seventynine said they had used opiates during the 24 hours prior to arrest and of these, 60 reported that the drug used was heroin. When those with past face-to-face contact were compared with those not previously seen, no significant differences were found in their treatment, diagnosis or self-reported use.

There were nine drug users arrested two or three times during the year and they therefore constituted 19 of the 92 cases (21\%). Seven of these had been seen by services face-to-face prior to the first arrest. The remaining two had not made contact with services, even by the time of their second arrest.

Only one alcohol misuser was arrested on more than one occasion and he had been in prior contact with services. Thirty of the 32 alcohol misusers were diagnosed as alcohol-intoxicated and the remaining two were prescribed benzodiazepines for alcohol withdrawal. There were no significant differences between those with past face-to-face contact and those without for demographic variables such as gender and type of accommodation.

\section{Clinical implications}

In this study, two-thirds of drug users had been referred to local treatment services prior to their arrest and 58\% had made face-to-face contact. Edmunds (1998) found that $48 \%$ of detained opiate users seen by three arrest referral schemes had been in prior contact with services, although they did not describe the nature of these contacts. Bennett (1998) interviewed detainees in five cities and found that $66 \%$ of those who described themselves as dependent on one or more drugs had received treatment from a variety of sources prior to arrest. In Cambridge, 59 subjects described themselves as dependent and $61 \%$ reported prior treatment, half at specialist drug-treatment services. Thus, most arrested drug misusers have had prior contact with services and our study indicates that the majority have made direct face-to-face contact. Consequently, for arrest referral schemes to be cost-effective, they must not only expedite referrals but also succeed in motivational enhancement.

There were no significant differences in reported consumption, diagnosis and treatment between those drug misusers with past face-to-face contact with services and the rest. However, those not in direct contact with services were more likely to be both homeless and male. Those living in transient accommodation or recently moved into the area were less likely to have engaged with local drug services. We were not able to record their length of stay in the city prior to arrest. Thirty-eight per cent of the sample were homeless or living in hostels and this reflected the problem of rough sleepers in Cambridge. The underrepresentation of women in the group without face-toface contact is more difficult to explain as there were no significant gender differences between the homeless and those in stable accommodation. One in five arrests were recidivists, in that they had been arrested on more than one occasion. Seventy-eight per cent had been in face-to-face contact with services prior to their first arrest. The high offending levels within this small cohort reflects the severity of their addiction and they are therefore a particularly challenging group to help re-engage in treatment.

The characteristics of this sample were very similar to another study of arrested drug users in London. Pearson et al (2000) found the mean age of 115 detainees to be 29 years, with the proportion of women at $28 \%$. In our sample, the mean age was 28 years and $27 \%$ were women. However, the proportion withdrawing from opiates differed. In the London study, only $19 \%$ of those seen by the police surgeon were diagnosed with withdrawal symptoms, whereas the proportion was $57 \%$ in our sample. Inversely, twice as many drug users were intoxicated in the London study. These differences were reflected in the treatment response. Only a third of the London sample were prescribed medication, but threequarters of the Cambridge drug users were treated at the police station. In both studies, a combination of dihydrocodeine and benzodiazepine was the usual approach.

The profile of drug users and service provision might differ across localities. Factors such as accessibility and waiting times for treatment may influence the proportion of arrested drug users not in contact with services. If the National Treatment Agency succeeds in establishing equitable services across the country, then providing drug users with information to facilitate referral to services may be a cost-effective intervention where waiting times 
original papers are reduced. However, this might not apply in areas with relatively easy access to services. The evidence base for motivational enhancement in the transient coercive setting of the police cell does not as yet exist (Miller, 1998). Another approach developing within the criminal justice field is the drug treatment and testing order. This is applied by the courts after conviction and would appear to be a more obvious means to influence motivation. Thus, the cost-effectiveness of these two approaches may vary in different geographical areas, depending on the local profile of arrested drug users and services. Rather than the current blanket approach, these criminal justice initiatives could be applied more selectively, with local research contributing to the decisions.

\section{References}

BENNETT,T. (1998) Drugs and Crime: MILLER, W. R. (1998) Why do People The Results of Research on Drug Testing ChangeAddictive Behaviour? The 1996 and Interviewing Arrestees. Home Office Research Study 183. David Archibald Lecture. Addiction, 93

EDMUNDS, M. (1998) Arrest Referral: Emerging Lessons from Research. Home Office Drugs Prevention PEARSON, R., ROBERTSON, G. \& GIBB, R. (2000) The identification and treatment of opiate users in police custody. Medicine, Initiative.

*Mervyn London Consultant Psychiatrist, Drug \& Alcohol Service, The Mill House, Brookfields Hospital, 351 Mill Road, Cambridge CB1 3DF,

Juan Canitrot Visiting Psychiatrist, Alvarez Hospital, Buenos Aires, Argentina, Adrian Dzialdowski Clinical Psychologist, Drug \& Alcohol Service, Brookfields Hospital, Cambridge, Robert Bates Specialist Registrar in Forensic Psychiatry, South Birmingham Mental HealthTrust,

Alan Gwynn General Practitioner and Police Surgeon, Brookfields Health Centre, Cambridge 